\title{
Detection of Delaminations in 3D Composites
}

\author{
E. Schnack*, S. Dimitrov, T.-A. Langhoff \\ Institute of Solid Mechanics \\ Karlsruhe University, D-76128 Karlsruhe, Germany. \\ eckart.schnack@imf.mach.uka.de
}

\section{SUMMARY}

There are several methods for inspecting material composites. One very well known one is the ultrasonic method, but the problem is that you normally obtain information only from the surface of these composites, e.g., methods of technical optics, making it necessary to analyze which layer contains the defect and how large it is. This knowledge is very important because arising from this defect comes a re-shifting of the threedimensional stress state, which can be very critical in construction. In this paper, we first present the original problem from which an iteration procedure is developed for solving this kind of problem and, subsequently, we show the complete theoretical structure for the identification together with numerical and experimental tests.

\section{INTRODUCTION}

In the past there have been several proposals to solve the problem of identification of cracks inside composites. You can find, among others, an introduction to the problem in the following papers: Alessandrini et al. /1/, Duduchava et al. /2/, Nishimura et al. 15/. Without lack of generality, let us consider the following geometrical outset for the problem of crack identification: given composite solid body with boundary

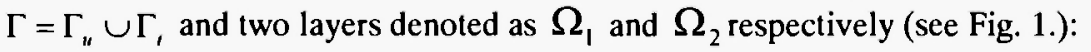

On the portion $\boldsymbol{i}_{::}$of the boundary are imposed displacement boundary conditions (i.e. DIRICHLET

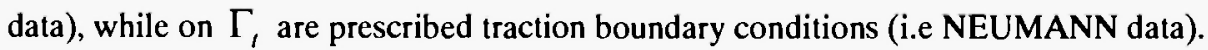

Here it is assumed $\Gamma_{u} \cap \Gamma_{i}=\varnothing$. Then, for the given displacement field $\mathbf{u}^{*}$ the objective function that should be minimized is simply the $L^{\bar{L}}$ norm measuring the distance between $\mathbf{u}^{*}$ and simulated data, i.e. 
$\left.\phi=\left\|\mathbf{u}^{*}-\tilde{\mathbf{u}}^{*}\right\|_{L^{2}\left(\Gamma_{u} \cup \Gamma_{t}\right)}=\sum_{\backslash \Gamma_{u} \cup \Gamma_{t}}\left|\mathbf{u}^{*}-\tilde{\mathbf{u}}^{*}\right|^{2} \mathrm{~d} \Gamma\right)^{1 / 2}$

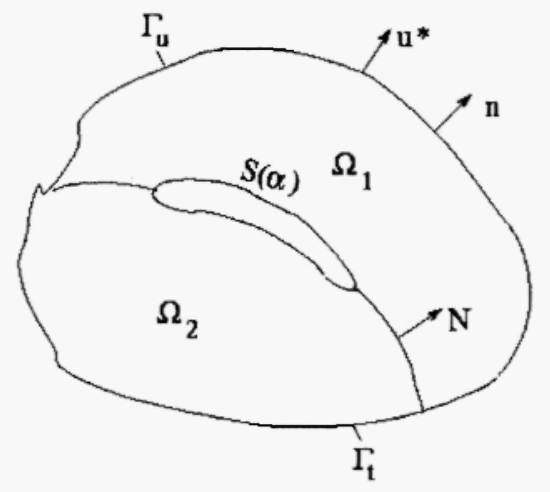

Fig. 1: Crack in a two-layered composite

As a result of minimization, we obtain a sequence of numerical solutions

$$
\overline{\mathbf{u}}_{k}^{*} \in\left\{\mathbf{u}_{k}^{*}\right\}_{k=1}^{n}
$$

In a more rigorous context, the identification problem for delamination can be formulated as follows: find a sequence of solutions (2) minimizing the functional (1):

$$
\min _{\tilde{\mathbf{u}}_{k}^{*}}\left\|\mathbf{u}^{*}-\ddot{\mathbf{u}}_{i k}^{i}\right\|_{L^{2}\left(\boldsymbol{\Gamma}_{u} \cup \Gamma_{t}\right)}^{2} \stackrel{\text { SQP }}{\longrightarrow}\left\{\alpha_{1}, \ldots, \alpha_{m}\right\}
$$

where the vector $\left\{\alpha_{1}, \ldots, \alpha_{m}\right\}$ determines the parametric description of the delaminating boundary $\mathrm{S}(\alpha)$ (see Fig. 1). The problem (3) allows Sequential Quadratic Programming Technique (SQP) for finding a feasible parameterization of the delamination surface. This is the principal problem we need to solve. Besides this traditional way, there exists an alternative way for solving this problem if we go back to the paper by Kozlov et al. $/ 4$.

The alternative way to solve the identification problem starts with the set of equations:

$$
\begin{aligned}
\mathcal{A} \mathbf{u} & =0 \quad \text { in } \Omega \\
\mathbf{u} & =\mathbf{u}^{*} \text { on } \Gamma_{u} \text { with } \Gamma_{u}=\Gamma \backslash \Gamma_{t} \\
\mathcal{T}(\nabla, \mathbf{n}) \mathbf{u} & =\mathbf{t}^{*} \text { on } \Gamma_{t}
\end{aligned}
$$


for which the corresponding variational problem reads as: find a function $\mathbf{u} \in H^{\prime}(\Omega)$ in the Sobolev space

$$
\left\{\mathbf{v} \in H^{1}(\Omega):\left.\mathbf{v}\right|_{\mathbf{r u}_{u}}=\mathbf{u}^{*}\right\}
$$

on which the functional

$$
F(\mathbf{v})=\int_{\Omega} a(\mathbf{v}, \mathbf{v}) \mathrm{d} \Omega-\int_{\Gamma_{1}} \mathbf{t}^{*} \mathbf{v} \mathrm{d} \Gamma
$$

attains its minimum. This solution trivializes also the differential equation (4) together with imposed boundary conditions.

In appropriate spaces we can formulate also the complementary problem

$$
\begin{aligned}
\mathcal{A} \mathbf{u} & =0 \quad \text { in } \Omega \\
\mathbf{u} & =\mathbf{u}^{* *} \text { on } \Gamma_{t} \\
\mathcal{T}(\nabla, \mathbf{n}) \mathbf{u} & =\mathbf{t}^{* *} \text { on } \Gamma_{u}
\end{aligned}
$$

Going further in the same direction we can state that both problem (4) as well as problem (7) are wellposed and possess unique solutions in $H^{1}(\Omega)$.

To determine the solution, we now introduce an iteration procedure. On the basis of (4) and (7) we have constructed an over-determined problem with DIRICHLET data. The same can be done analogously for the case of NEUMANN data. "Over-determined", means that on the boundary $\Gamma_{u}$ are prescribed as DIRICHLET as well as NEUMANN data. On this basis we can start an iterative procedure with the initial approximation:

$$
\begin{aligned}
& \mathcal{A} u^{(0)}=0 \text { in } \Omega \\
& \mathbf{u}^{(0)}=\tilde{\mathbf{u}}^{*} \text { on } \Gamma_{u} \\
& \mathcal{T}(\nabla, \mathbf{n}) \mathbf{u}^{(0)}=\mathbf{t}^{(0)} \text { on } \Gamma_{t}
\end{aligned}
$$

If we assume further that $\mathbf{u}^{(2 n)}$ is already constructed, then $\mathbf{u}^{(2 k+n)}$ must solve

$$
\begin{aligned}
& \mathcal{A u}_{\mathbf{u}^{(2 \ell+1)}}=0 \text { in } \Omega \\
& \mathbf{u}^{(2 k+1)}=\mathbf{u}^{(2 k)} \text { on } \Gamma_{t} \\
& \mathcal{T}(\nabla \cdot \mathbf{n}) \mathbf{u}^{(2 k+1)}=\dot{\mathrm{t}}^{*} \text { on } \Gamma_{u}
\end{aligned}
$$


Now, having $\mathbf{u}^{(2 k+1)}$ we have to solve the problem for $\mathbf{u}^{((2 k+1)+1)}$

$$
\begin{aligned}
\mathcal{A} \mathbf{u}^{((2 k+1)+1)} & =0 & & \text { in } \Omega \\
\mathbf{u}^{((2 k+1)+1)} & =\mathbf{u}^{*} & & \text { on } \Gamma_{u} \\
\mathcal{T}(\nabla, \mathbf{n}) \mathbf{u}^{((2 k+1)+1)} & =\mathcal{T}(\nabla, \mathbf{n}) \mathbf{u}^{(2 k+1)} & & \text { on } \Gamma_{t}
\end{aligned}
$$

In abstract form this system was first proposed and analyzed by Kozlov et al. $/ 4 /$.

\section{The Reconstruction Algorithm}

First, one must decide on the experimental method to obtain the information for the displacements on the surface. In addition, we must know which inspection method gives us the best sensitivity of a non-destructive testing technique. Gros et al. /3/ compared the visual method (naked-eye), the shearography method, the ultrasonic method (it means the C-scan method), the eddy current method, the infrared thermography method and the $\mathrm{x}$-ray radiography method. From all these together, you can see that from the automatic methods, the shearography method is the best because the minimal impact energy can be detected which is, apart from the automatic techniques, only possible for the visual method by the naked-eye.

With this information, we now define the reconstruction technique. The main idea, at first, is the decomposition of the problem into two Cauchy sub-problems (see Fig. 2)

This decomposition can be formally represented with two sets of equations:

$$
\begin{aligned}
& \mathcal{A}_{1} \boldsymbol{u}_{1}=\mathbf{0} \text { in } \Omega_{1}, \\
& \boldsymbol{u}_{1}=\boldsymbol{u}_{1}^{*} \text { on } \Gamma_{1} \text {, } \\
& \mathcal{T}_{1}(\nabla, n) u_{1}=t_{1}^{*} \text { on } \Gamma_{1} \text {, } \\
& \mathcal{A}_{2} \boldsymbol{u}_{2}=\mathbf{0} \quad \text { in } \Omega_{2} \text {. } \\
& \boldsymbol{u}_{2}=\boldsymbol{u}_{2}^{*} \text { on } \Gamma_{2} \text {. } \\
& \mathcal{T}_{2}(\Gamma, \boldsymbol{n}) \boldsymbol{u}_{2}=\boldsymbol{t}_{2}^{*} \text { on } \Gamma_{2} \text {. }
\end{aligned}
$$

The systems (11) and (12) must be solved, so the displacements $\mathbf{u}_{1}$ and $\mathbf{u}_{2}$ at the given interface $\Gamma$ to be obtained. From that we get the region of the crack $\mathrm{S}(\alpha)$ as the support of the difference of the projections of displacements to the normal $(\mathrm{N})$ :

$$
S=\left.\operatorname{supp}\left[\mathbf{u}_{\mathbf{N}}\right]\right|_{\mathbf{r}}
$$




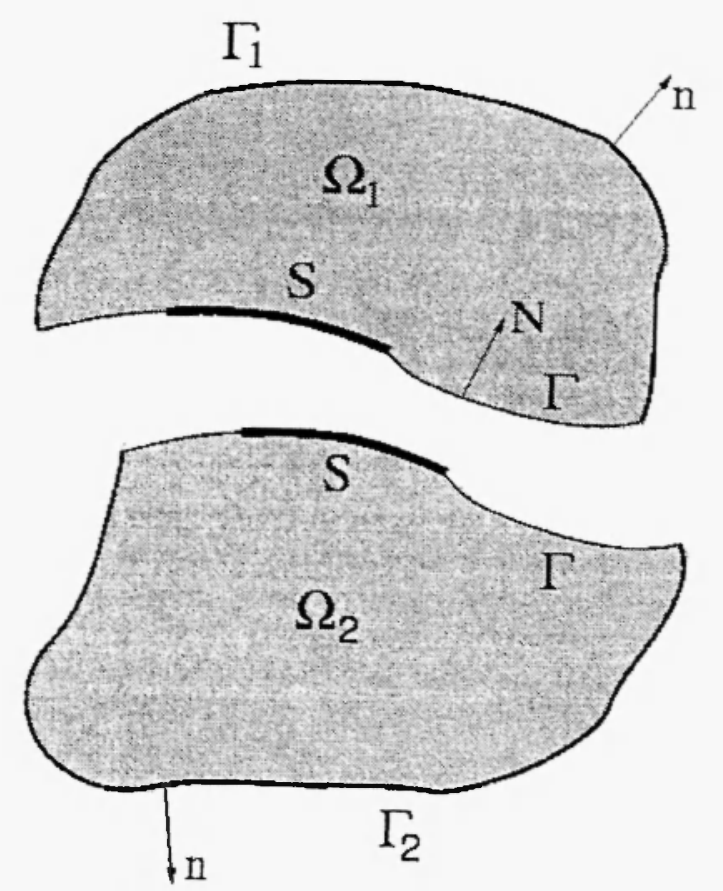

Fig. 2: Decomposition in two Cauchy sub-problems

The algorithmic description of the iteration procedure is as follows:

1. Initial solution

$$
\boldsymbol{u}_{1}^{(0)}=u_{2}^{(0)}=\boldsymbol{u}^{(0)} \text { on } \Gamma \text {. }
$$

2. Then we are beginning with iteration step $k=1$ :

$$
\begin{aligned}
\mathcal{A}, \boldsymbol{u}_{i}^{(2 k-1)} & =0 \text { in } \Omega_{1}, \\
\boldsymbol{u}_{2}^{(2 k-1)} & =\boldsymbol{u}_{i}^{(2 h-2)} \text { on } \Gamma, \\
\mathcal{T}_{i}(\nabla, n) \boldsymbol{u}_{i}^{(2 k-1)} & =\boldsymbol{t}^{*} \text { on } \Gamma_{1} .
\end{aligned}
$$

to determine

$$
\mathbf{u}^{(2 k-1)} \text { in } \Omega_{i}
$$

for $t=1,2$. 
3. In the next iteration-step $\mathrm{k}$ we have to solve

$$
\begin{aligned}
\mathcal{A}_{i} \boldsymbol{u}_{i}^{(2 k)} & =0 \text { in } \Omega_{,}, \\
\boldsymbol{u}_{i}^{(2 k)} & =u^{*} \text { on } \Gamma, \\
\mathcal{T}_{i}(\nabla . N) \boldsymbol{u}_{i}^{(2 k)} & =t_{i}^{(2 k-1)} \text { on } \Gamma .
\end{aligned}
$$

where

$$
\mathbf{t}_{i}^{(2 k-1)}=\mathcal{T}_{i}(\nabla, \mathbf{n}) \mathbf{u}_{i}^{(2 k-1)} \mid \boldsymbol{\Gamma}
$$

to determine

$$
\mathbf{u}_{i}^{(2 k)} \text { in } \Omega \text {, }
$$

for $\iota=1,2$.

4. We have to calculate the approximate displacement jump across the interface $\Gamma$

$$
\left.[\mathbf{u}]^{(2 k)}\right|_{\mathbf{r}}=\left.\mathbf{u}_{1}^{(2 k i)}\right|_{\mathbf{r}}-\left.\mathbf{u}_{2}^{(2 k)}\right|_{\mathbf{r}}
$$

5. After that we have to determine the approximate crack domain $5_{\varepsilon}^{(2,)} \subset \Gamma$ by choosing $\varepsilon^{(2 k)}$

$$
S_{\vec{q}}^{(2 k)}=\left\{\mathbf{x} \in \Gamma:\left[\mathbf{u}^{(2 k)}\right] \cdot N>\varepsilon^{(2 k \cdot)}\right\}
$$

6. Increase $k \rightarrow k+1$ and return to step 2 until the given stopping criterion is fulfilled.

It is very important to note that there exists an extension of this method to a three boundary variant, which means that we do not have all the data of the over-determined system (see Weikl et al. /6/). It is very nice that we have convergence of this method, in principle, and that we can fulfill the regularizing properties (see Weikl et al. /6/).

Since we are working with Finite Elements to numerically solve the mixed boundary value problems (15) and (16), we have, moreover, to consider the parameter of the mesh-size. On the basis of the Hausdorff metric, an additional convergence theorem (see Weikl et al. /6/) is obtained. 


\section{Numerical Results}

We are analyzing in the following an 8-layer laminate where we assume that the sequence of orientations of the different layers is given by $[+45,-45,0,90,90,0,-45,+45]$. The mesh for the laminate contains 9126 nodes and 5568 elements (see Fig. 3):

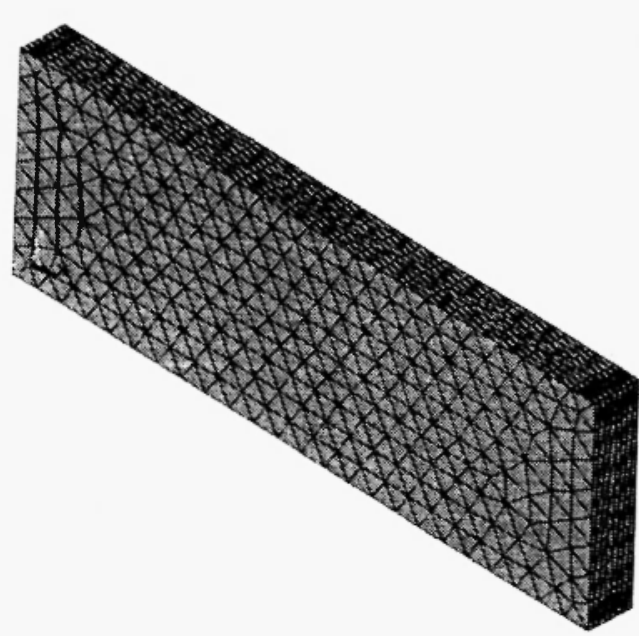

Fig. 3: Mesh of the laminate containing 8 layers, 9126 nodes and 5568 elements

This special specimen is loaded by tension and you can see a typical mesh on the surface in Fig. 4:

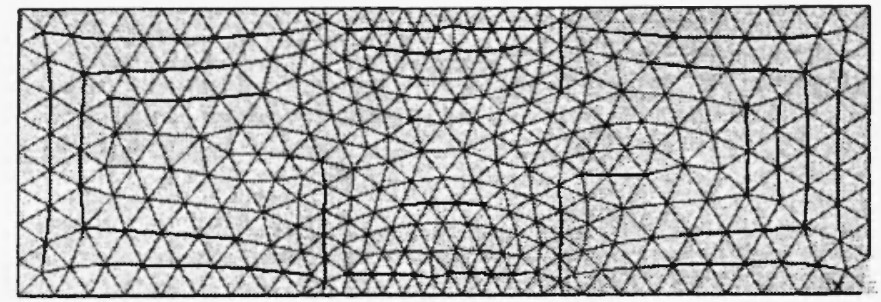

Fig. 4: Surface mesh on $\Gamma$

The 3-boundary iteration process with experimental data from the out-of-plane displacements is used.

After 4 iterations (see Fig. 5 a)) the results can already quite well detect the region of the crack. After 10 iterations (see Fig. 5 b)), no significant change of the information can be stated. So the proposed method shows a fast convergence and can really be used to detect defects in laminate structures. 


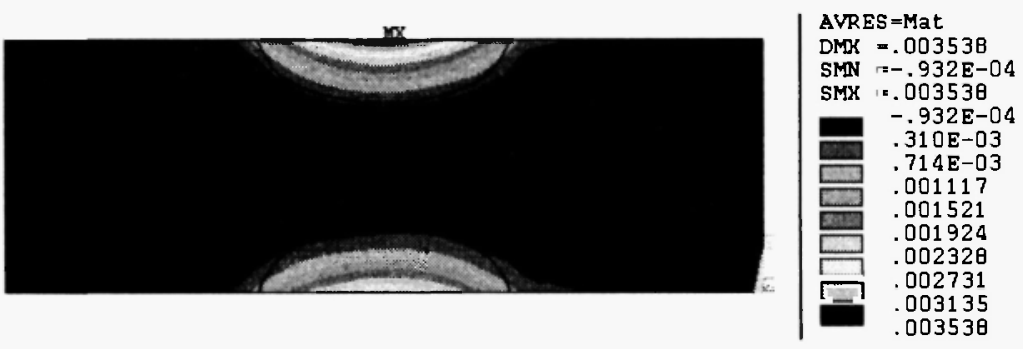

4 iterations

Fig. 5: a): Numerical results in comparison with experimental data (4 iterations). The plotted values correspond to the $\mathbf{z}$-direction of the displacements.

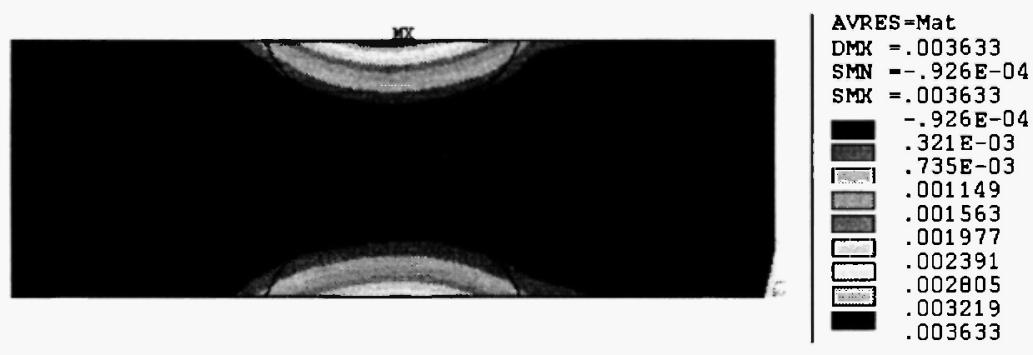

10 iterations

Fig. 5: b): Numerical results in comparison with experimental data (10 iterations). The plotted values correspond to the z-direction of the displacements.

\section{CONCLUSIONS}

The paper represents a combined empirical-numerical procedure for detection of defects inside of composites, where, besides the classical methods, a special iteration technique is utilized and extended. Because of the given regularization property, results are obtained in a robust way. The simplicity and reliability of the method allow its direct implementation in engineering practice.

\section{REFERENCES}

1. G. Alessandrini, E. Beretta and S. Vessella, Determining linear cracks by boundary measurements lipschitz stability, SIAM J. Math. Anal. 27, 261-375 (1996).

2. R. Duduchava, A.M. Sändig and W.L. Wendland, Interface cracks in anisotropic composites, Mathem. 
Meth. Appl. Sci. 22, 1413-1446 (1999).

3. X.E. Gros, K. Takahashi and M.-A. De Smet, On the efficiency of current NDT methods for impact damage detection and quantification in thermoplastic toughened CFRP materials, NDT. Net, 4, (1999).

4. V.A. Kozlov, V.G. Maz'ya and A.V. Fomin, An iterative method for solving the Cauchy problem for elliptic equations, Comput. Maths. Math. Phys. 31, 45-52 (1991).

5. N. Nishimura and S. Kobayashi, A boundary integral equation method for an inverse problem related to crack detection, Int. J. Num. Meth. Engng. 32, 1371-1387 (1990).

6. W. Weikl, H. Andrä and E. Schnack, An alternating iterative algorithm for the reconstruction of internal cracks in a 3-D solid body, Inverse Problems 17, 1957-1975 (2001). 
